\title{
Safety and preliminary efficacy of deep transcranial magnetic stimulation in MS-related fatigue
}

\section{OPEN}

Gunnar Gaede, MD

Marina Tiede, MD

Ina Lorenz, MD

Alexander U. Brandt, MD

Caspar Pfueller, MD

Jan Dörr, MD

Judith Bellmann-Strobl,

MD

Sophie K. Piper, PhD

Yiftach Roth, PhD

Abraham Zangen, PhD

Sven Schippling, MD

Friedemann Paul, MD

Correspondence to

Dr. Paul:

friedemann.paul@charite.de

\section{ABSTRACT}

Objective: To conduct a randomized, sham-controlled phase I/Ila study to evaluate the safety and preliminary efficacy of deep brain $\mathrm{H}$-coil repetitive transcranial magnetic stimulation (rTMS) over the prefrontal cortex (PFC) and the primary motor cortex (MC) in patients with MS with fatigue or depression (NCT01106365).

Methods: Thirty-three patients with MS were recruited to undergo 18 consecutive rTMS sessions over 6 weeks, followed by follow-up (FU) assessments over 6 weeks. Patients were randomized to receive high-frequency stimulation of the left PFC, MC, or sham stimulation. Primary end point was the safety of stimulation. Preliminary efficacy was assessed based on changes in Fatigue Severity Scale (FSS) and Beck Depression Inventory scores. Randomization allowed only analysis of preliminary efficacy for fatigue.

Results: No serious adverse events were observed. Five patients terminated participation during treatment due to mild side effects. Treatment resulted in a significant median FSS decrease of 1.0 point $(95 \% \mathrm{Cl}[0.45,1.65])$, which was sustained during FU.

Conclusions: $\mathrm{H}$-coil rTMS is safe and well tolerated in patients with MS. The observed sustained reduction in fatigue after subthreshold MC stimulation warrants further investigation.

ClinicalTrials.gov identifier: NCT01106365.

Classification of evidence: This study provides Class III evidence that rTMS of the prefrontal or primary MC is not associated with serious adverse effects, although this study is underpowered to state this with any precision. Neurol Neuroimmunol Neuroinflamm 2018;5:e423; doi: 10.1212/ NXI.0000000000000423

\section{GLOSSARY}

ANOVA $=$ analysis of variance; $\mathbf{B D I}-\mathbf{I A}=$ Beck Depression Inventory $\mathbf{I A}$; $\mathbf{B L}=$ baseline; EDSS = Expanded Disability Status Scale; FSMC = Fatigue Scale for Motor and Cognitive Functions; FSS = Fatigue Severity Scale; 9 -HPT = 9 Hole Peg Test; IQR = interquartile range; $\mathbf{M C}$ = motor cortex; $\mathbf{M D D}$ = major depressive disorder; $\mathbf{M F I S}=$ Modified Fatigue Impact Scale; $\mathbf{M S F C}=$ multiple sclerosis functional composite; PASAT $=$ Paced Auditory Serial Addition Test; PFC $=$ prefrontal cortex; $\mathbf{R M T}=$ resting motor threshold; $\mathbf{r T M S}$ = repetitive transcranial magnetic stimulation; $\mathbf{S A E}=$ serious adverse event; T25FW = timed 25-ft walk.

MS is the most common autoimmune inflammatory and neurodegenerative disease of the CNS. ${ }^{1}$ Fatigue is one of the most frequent symptoms experienced in MS, affecting up to $90 \%$ of patients. ${ }^{2}$ Although MS fatigue contributes to poor health-related quality of life ${ }^{3}$ and is a major factor in disease-related unemployment, ${ }^{4}$ its etiology has not yet been fully elucidated, and efficacious treatment options are scarce. Neuroimaging studies suggest that structural and functional connectivity alterations, particularly to interconnections between the basal ganglia

Supplemental data at http://links.Iww.com/ NXI/A2

\footnotetext{
From the NeuroCure Clinical Research Center (G.G., A.U.B., C.P., J.D., J.B.-S., S.K.P., F.P.), Center for Stroke Research Berlin (CSB) (S.K.P.), Experimental and Clinical Research Center (J.B.-S., F.P.), and Clinical and Experimental Multiple Sclerosis Research Center (F.P.), Department of Neurology, Charité-Universitätsmedizin Berlin, Germany; Institute of Neuroimmunology and Multiple Sclerosis (M.T., I.L., S.S.), University Medical Centre Hamburg-Eppendorf, Germany; Department of Life Sciences (Y.R., A.Z.), Ben-Gurion University, Beer-Sheva, Israel; and MaxDelbrück Center for Molecular Medicine (F.P.), Berlin, Germany. G.G. and C.P. are now with St. Joseph Krankenhaus-Berlin-Weissensee, Germany. S.S. is now with Neuroimmunology and Multiple Sclerosis Research, Department of Neurology, University Hospital Zurich and University of Zurich, Switzerland.

Funding information and disclosures are provided at the end of the article. Go to Neurology.org/nn for full disclosure forms. The Article Processing Charge was funded by the authors.

This is an open access article distributed under the terms of the Creative Commons Attribution-NonCommercial-NoDerivatives License 4.0 (CC BY-NC-ND), which permits downloading and sharing the work provided it is properly cited. The work cannot be changed in any way or used commercially without permission from the journal.
} 
and the prefrontal cortex (PFC), the posterior cingulate cortex and cortical motor areas, may contribute to fatigue in MS. ${ }^{5-9}$

A potential treatment of functional connectivity impairment is noninvasive neuromodulation by means of repetitive transcranial magnetic stimulation (rTMS). Highfrequency rTMS of the left PFC has been demonstrated as safe and efficacious in major depressive disorder (MDD). ${ }^{10}$ Moreover, 5-Hz rTMS applied to the primary motor cortex (MC) improved lower limb spasticity ${ }^{11}$ and bladder dysfunction ${ }^{12}$ in patients with MS.

rTMS using $\mathrm{H}$-coils, a technology developed several years later, allows brain stimulation 3 times deeper than that of standard figure-of-eight coils at the expense of focality. ${ }^{13,14}$ It has also been widely proven as safe and well tolerated, including in a study of healthy volunteers ${ }^{15}$ and, in the form of high-frequency stimulation of the left PFC, as effective and safe treatment of MDD. ${ }^{16-18}$ PFC and MC stimulation directly targets circuits for which alterations in fatigue were reported, ${ }^{5-9}$ and PFC stimulation is supported by the high overlap between fatigue and depressive symptoms. ${ }^{19} \mathrm{MC}$ stimulation, on the other hand, may also lead to an additional improvement of fatigue via reduction of spasticity. ${ }^{11}$

Against this background, we conducted a randomized, sham-controlled phase I/IIa pilot study to evaluate the safety, tolerability, and preliminary efficacy of deep brain H-coil rTMS over the left PFC and MC as treatment of fatigue and depression in MS.

METHODS Study design and participants. The study was designed as a prospective, randomized, semi-blinded, sham-controlled phase I/IIa pilot study involving $2 \mathrm{MS}$ centers (NeuroCure Clinical Research Center, Charité-Universitätsmedizin Berlin, Germany, and the Institute of Neuroimmunology and Multiple Sclerosis research at the University Hospital Hamburg Eppendorf, Hamburg, Germany). Primary end point in this study was safety of rTMS stimulation in patients with MS. The study design and workflow are presented in figure 1. Patients underwent an initial screening/baseline (BL) visit 4 weeks before intervention, from which all BL characteristics were derived and a second scheduling visit to confirm study inclusion or exclusion and to schedule an appointment for commencement of the intervention 2 weeks later. Patients were then randomized into 3 groups on the day of treatment initiation, followed by 6 weeks of intervention. After the treatment phase, patients were followed up every 2 weeks over 6 weeks (follow-ups: FU1, 2, and 3). Recruitment period was from May 2010 to March 2011. This pilot trial was performed without sample size calculation; randomization of 33 patients was planned. Recruitment was stopped regularly after randomizing 33 patients. The study provides Class III evidence.

Inclusion criteria were diagnosis of MS according to the 2005 revised McDonald criteria, ${ }^{20}$ either a score of $\geq 4$ on the Fatigue Severity Scale $(F S S)^{21}$ or a score of $\geq 12$ on the Beck Depression Inventory IA (BDI-IA), ${ }^{22}$ age (18-65 years), relapse-free for at least 3 months, and free of steroid treatment within 30 days prior to inclusion, Expanded Disability Status Scale (EDSS) between 0.0 and 6.0, stable immunotherapy or antidepressant therapy for at least 3 months if applicable, and highly effective methods of contraception for females. Exclusion criteria were history of seizures (personal or family), history of stroke, head injury, metal fragments in the head, implanted devices such as cardiac pacemakers, cochlear implants, medical pumps, alcohol or drug abuse, pregnancy, comedication with neuroleptics or tricyclic antidepressants, increased intracranial pressure, bipolar affective disorder, significant neurologic, psychiatric, cardiovascular, hepatic, renal, gastrointestinal, metabolic, or other systemic comorbidities.

Abortion criteria were personal wish of the participant, any relapse, exacerbation of depressive symptoms including suicidality and suicide attempt, comedication with tricyclic antidepressant, pregnancy, safety concerns, noncompliance, loss to FU, and missing more than 3 treatment sessions.

Standard protocol approvals, registrations, and patient consents. The study was registered at clinicaltrials.gov as NCT01106365 and was approved by the local ethics committees of the Charité-Universitätsmedizin Berlin and by the Hamburg Board of Physicians. It was conducted in accordance with the Declaration of Helsinki and the guidelines of the International Conference on Harmonisation of Good Clinical Practice. All participants provided written informed consent.

Randomization and masking. Patients were randomized in a 1:1:1 ratio to receive H6 coil rTMS over the left prefrontal cortex ("PFC" group), sham rTMS over the same area ("PFC sham" group), or H10 coil rTMS over the primary motor cortex bilaterally ("MC" group). For randomization, Brainsway Ltd. created individual treatment cards, which guaranteed blinding. ${ }^{18}$ Patients were enrolled in each center and assigned to groups using the treatment cards by independent operators. The PFC sham condition induced superficial magnetic sensations comparable to the 2 therapeutic stimulation conditions, preventing patients from distinguishing sham treatment by sensation or hearing. For PFC and PFC sham conditions, both patients and operators were blinded (double-blinded condition). For the MC stimulation, a different coil without extra sham function was used, thus operator blinding was not possible. Thus, TMS operators were aware of the MC stimulation condition, whereas the patients as well as interviewing and examining neurologists were not (single blinded).

Diagnostic procedures. During treatment and follow-up, a trained neurologist, who was masked to treatment allocation, conducted a weekly and biweekly clinical interview and physical examination, respectively, of each patient to assess safety and tolerability. Moreover, patients were advised to contact the study centers by phone or present to emergency services in case of severe adverse events. We a priori defined any seizure or MS relapse as serious adverse events (SAEs). Second, reports of the previous week's symptoms were assessed to calculate the $\mathrm{FSS}^{21}$ and the BDI-IA ${ }^{22}$ questionnaire as indicator of preliminary clinical efficacy. Patients with an FSS score of $\geq 4$ were classified as fatigued..$^{21,23}$ Patients with a BDI score of $\geq 12$ were classified as depressed. ${ }^{22}$ 
Figure 1 Patient flow diagram

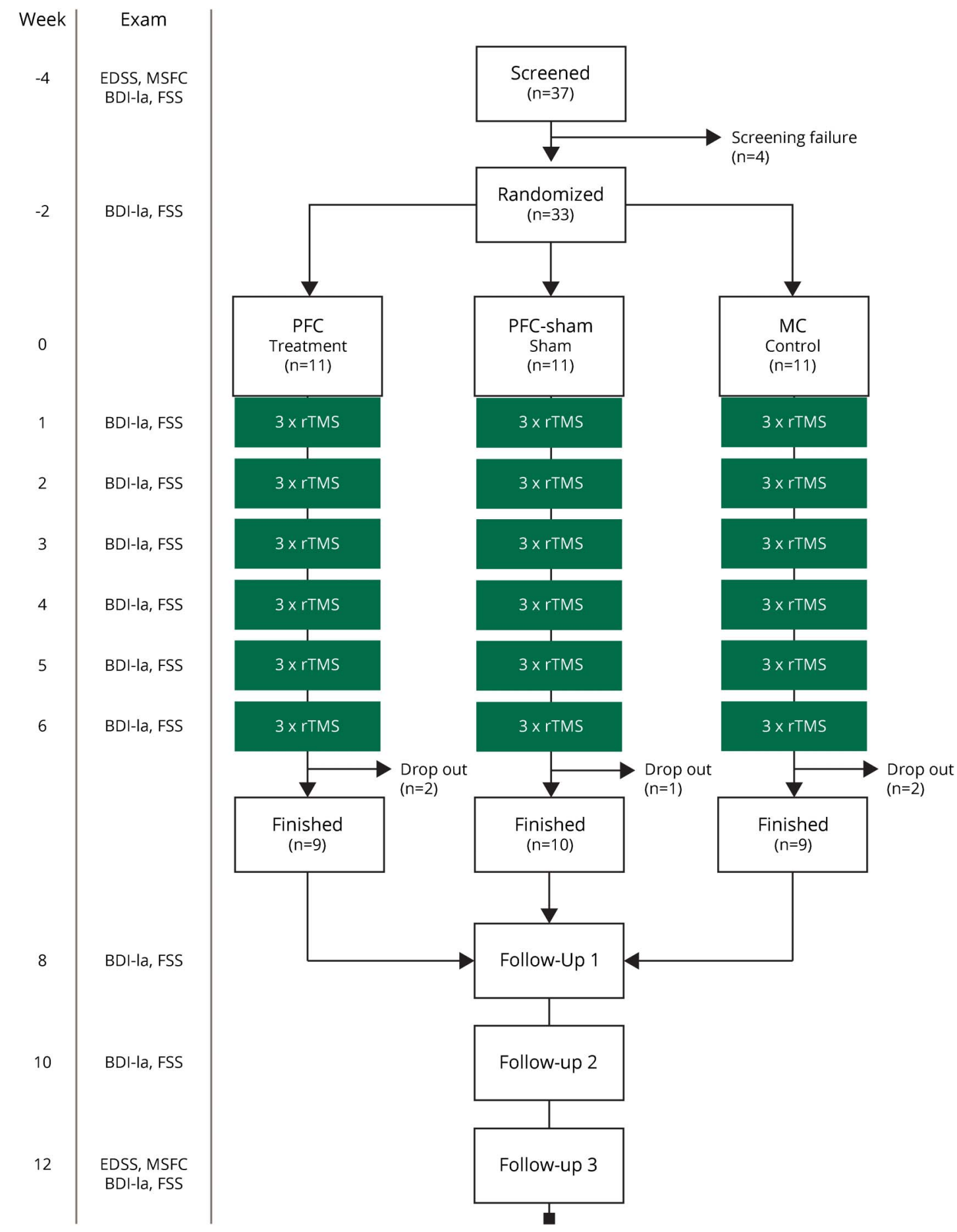

Patient flow in this interventional study. The column on the left side lists the week relative to the first day of intervention. The second column lists the diagnostic parameters assessed at the visits. The column on the right side depicts the patient flow including randomization in the 3 treatment groups. BDI-IA = Beck Depression Inventory IA; EDSS = Expanded Disability Status Scale; FSS = Fatigue Severity Scale; MC = motor cortex; MSFC = Multiple Sclerosis Functional Composite; PFC = prefrontal cortex; rTMS = repetitive transcranial magnetic stimulation.

In addition, the $\mathrm{EDSS}^{24}$ and the multiple sclerosis functional composite (MSFC), including the timed 25-ft walk (T25FW) test, the 9-Hole Peg Test (9-HPT), and the Paced Auditory Serial Addition Test (PASAT), were assessed at screening and in the final FU visit. ${ }^{25}$ MSFC Z-scores were calculated from T25FW, 9-HPT, and PASAT results with reference to the screening examination including all patients. ${ }^{25}$
Transcranial magnetic stimulation. rTMS was performed by 3 operators using $\mathrm{H}$-coils (Brainsway Ltd., Jerusalem, Israel) connected to a Magstim Rapid ${ }^{2}$ stimulator (Magstim, Spring Gardens, United Kingdom). Each participant received 3 rTMS sessions per week over a period of 6 weeks (total of 18 treatments) followed by a 6-week FU period comprising biweekly clinical assessments and questionnaires. 
Bilateral MC stimulation in the "MC" group was performed with the $\mathrm{H} 10$ coil, designed for the activation of hand or leg MC. We applied 40 trains of bihemispherical stimulation of the MC, with bursts of 20 stimuli with an intertrain interval of 20 seconds at a frequency of $5 \mathrm{~Hz}$ (intensity of $90 \%$ resting motor threshold [RMT]) with a total number of 800 stimuli and a total duration of 16 minutes, positioning the center of the coil mid-sagittally over the primary MC.

Real left PFC stimulation in the "PFC" group was performed with the left $\mathrm{H} 6$ coil, designed for the activation of superficial and deep left PFC structures. To determine the individual RMT, the coil center was positioned over the motor hand area, and contralateral motor responses of the first dorsal interosseous muscle were recorded. Individual RMT was defined as the minimum intensity that evoked a potential of $50 \mu \mathrm{V}$ in 5 of 10 consecutive stimuli according to current international recommendations. ${ }^{26}$ The coil was positioned $5 \mathrm{~cm}$ anteriorly to the left motor hot spot, parallel to the sagittal suture of the skull in order to provide stimulation of the left PFC region. We applied a total of 50 trains (intensity of $120 \%$ of RMT) in each session, with a duration of 2 seconds at a frequency of $18 \mathrm{~Hz}$ repeating at 20-second intervals (total number of stimuli per session: 1,800; duration: 18 minutes).

The H6 coil but not the $\mathrm{H} 10$ coil included a sham function. Superficial skin stimulation with a sham function, which led to a comparable sound and sensation like real TMS, was ensured, but not stimulation of the brain. For sham stimulation, the coil was placed identically to the real PFC stimulation condition. Fifty trains of superficial stimulation of the skin were applied at the same frequency $(18 \mathrm{~Hz})$ and duration $(2$ seconds) at 20 -second intervals, as in the PFC stimulation paradigm (total number of stimuli per session: 1,800; duration: 18 minutes).
Statistical analysis. Results are reported as frequencies and percentages, median, and interquartile range (IQR), depending on the scale of the data. Baseline differences between PFC, PFC sham, and $\mathrm{MC}$ groups including all patients completing the trial were analyzed with nonparametric Kruskal-Wallis tests for ordinal and continuous data and with Fisher-Freeman-Halton exact tests for categorical data (table 1).

Clinical outcomes were FSS, BDI-IA, EDSS, and MSFC data assessed at BL, FU1, and FU3 visits (for FSS and BDI-IA) or FU3 (for EDSS and MSFC). FU1 FSS scores were not collected for 3 PFC, 1 MC-, and 1 PFC sham-stimulated patients, and BDI data for 2 PFC, 1 MC-, and 1 PFC sham-stimulated patients. Here, data from the next FU visit were used. For 1 PFC- and $1 \mathrm{MC}-$ stimulated patient, the third FU score had to be used. Patients whose FU1 data were replaced with FU3 data were excluded in the preliminary sustainability analysis, comparing the first and last FU data. We analyzed preliminary clinical efficacy and sustainability in an exploratory analysis using nonparametric (rank based) analysis of variance (ANOVA)-like computation of longitudinal data in factorial settings (R-package "nparLD"). The Wilcoxon signed-rank test was used for pairwise post hoc analysis of changes over time within each treatment group. The analysis of potential effects on fatigue included only those patients who were classified as fatigued (FSS $\geq 4.0$ ) at BL. Distribution of patients classified as depressed (BDI-IA $>12.0$ ) was uneven between the groups (table 1). We, therefore, abstained from further analyzing effects on depressive symptoms. To account for potential differences in fatigue and depression at $\mathrm{BL}$ between groups as potential confounders, we additionally performed a nonparametric (rank-based) ANOVA-like analysis for FSS score at FU1 visit, with adjustment for FSS and BDI values at BL.

\begin{tabular}{|c|c|c|c|c|c|}
\hline \multirow[t]{2}{*}{ Cohort ove } & fiew at baseline & & & & \\
\hline & & PFC & PFC Sham & MC & p Value ${ }^{a}$ \\
\hline Condition & & Treatment & Sham & Treatment & \\
\hline Patients & $\mathrm{n}$ & 9 & 10 & 9 & \\
\hline Disease course & RRMS/SPMS & $8 / 1$ & $9 / 1$ & $9 / 0$ & 1.000 \\
\hline Sex & $\mathrm{M} / \mathrm{F}$ & $3 / 7$ & $2 / 7$ & $1 / 8$ & 0.845 \\
\hline Age, y & Median (IQR) & 47 (32 to 51 ) & 41 (39 to 45 ) & 46 (42 to 48 ) & 0.323 \\
\hline Time since diagnosis, mo & Median (IQR) & 46 (37 to 110 ) & 67 (38 to 224) & 187 (91 to 258 ) & 0.048 \\
\hline EDSS & Median (IQR) & 2.5 (2.0 to 3.0$)$ & 3.0 (2.5 to 3.0$)$ & 2.5 (2.5 to 3.5 ) & 0.719 \\
\hline T25FW, s & Median (IQR) & 7.2 (6.1 to 9.5$)$ & 9.6 (6.5 to 11.7$)$ & 8.1 (6.3 to 8.7 ) & 0.365 \\
\hline 9-HPT dom., s & Median (IQR) & 20.0 (18.7 to 21.0) & 19.4 (17.5 to 23.6 ) & 18.9 (18.5 to 21.1$)$ & 0.916 \\
\hline 9-HPT ndom., s & Median (IQR) & 20.0 (19.5 to 22.7 ) & 20.3 (18.5 to 22.8) & 20.9 (19.3 to 21.9) & 0.864 \\
\hline PASAT, /60 & Median (IQR) & 56 (53 to 59 ) & 45 (40 to 48 ) & 51 (44 to 53 ) & 0.102 \\
\hline MSFC-Z & Median (IQR) & 0.5 (0.2 to 1.0$)$ & $-0.1(-0.6$ to 0.0$)$ & $0.0(-0.1$ to 0.4$)$ & 0.131 \\
\hline BDI-IA & Median (IQR) & 22.0 (19.0 to 26.0 ) & 14.0 (13.0 to 21.0$)$ & $12.0(9.0$ to 13.0$)$ & 0.002 \\
\hline BDI-IA >12 & Yes/no & $9 / 0$ & $8 / 2$ & $4 / 5$ & 0.023 \\
\hline Antidepressant Tx & Yes/no & $5 / 4$ & $5 / 5$ & $1 / 8$ & 0.119 \\
\hline FSS & Median (IQR) & 6.2 (5.3 to 6.3 ) & $6.0(4.6$ to 6.1$)$ & $6.0(5.6$ to 6.4$)$ & 0.501 \\
\hline FSS $\geq 4.0$ & Yes/no & $9 / 0$ & $9 / 1$ & $9 / 0$ & 1.000 \\
\hline
\end{tabular}

Abbreviations: 9-HPT = 9-hole peg test of the dominant hand; 9-HPT ndom. = 9-Hole Peg Test of the nondominant hand; BDI-IA = Beck Depression Inventory IA; EDSS = Expanded Disability Status Scale; FSS = Fatigue Severity Scale; IQR = interquartile range (25th and 75th percentiles); $M C=$ motor cortex MSFC = multiple sclerosis functional composite; PASAT = paced auditory serial additions test; $\mathrm{PFC}=$ prefrontal cortex; $\mathrm{RRMS}=$ relapsing-remitting MS; $r$ TMS = repetitive transcranial magnetic stimulation; SPMS = secondary progressive MS; T25FW = Timed 25-ft walk.

${ }^{a}$ For categorical data derived from Fisher exact tests with Freeman-Halton extension, all other data from Kruskal-Wallis tests. Significant $p$ values are printed in bold. 
Statistical analyses were performed using IBM SPSS Statistics software version 23 (IBM Corp., Armonk, NY), SAS 9.4 software (SAS Institute Software GmbH, Vienna, Austria), and R software version 3.2.4. ${ }^{27}$ All tests were 2 sided, and statistical significance was determined at an $\alpha$ level of 0.05 . Exploratory analyses had no previous sample size calculation, and no adjustment for multiple comparisons was applied.

RESULTS Patients. Thirty-seven patients with MS were assessed for eligibility. Four patients did not meet the inclusion criteria (figure 1). Of the remaining 33 patients, 5 dropped out before completion. Twenty-eight patients completed the treatment and FU phases. Detailed BL demographic and clinical data are presented in table 1 . Eleven patients were on stable antidepressant therapy (5 citalopram, 2 fluoxetine, 2 venlafaxine, 1 sertraline, and 1 St. John wort [Hypericum perforatum]). Two patients in the MC group received symptomatic treatment with modafinil. Baseline data were similar across groups for disease course, age, sex, EDSS, and MSFC (table 1).

Safety and tolerability. No SAE were observed in any group; however, known trigeminal neuralgia intensified in 1 PFC sham patient. Twenty-five of $28 \mathrm{pa}-$ tients reported at least 1 adverse event during the entire treatment period, while 1 sham patient and 2 patients with MC did not (table 2). The adverse event

Table 2 Adverse events

\begin{tabular}{|c|c|c|c|c|c|c|c|c|}
\hline \multirow[b]{2}{*}{ Reported events } & \multicolumn{2}{|c|}{ Total } & \multicolumn{2}{|c|}{ PFC } & \multicolumn{2}{|c|}{$\begin{array}{l}\text { PFC } \\
\text { Sham }\end{array}$} & \multicolumn{2}{|c|}{ MC } \\
\hline & $\mathrm{n}$ & $\%$ & $\mathrm{n}$ & $\%$ & $\mathrm{n}$ & $\%$ & n & $\%$ \\
\hline Increased headache during treatment & 3 & 11 & 2 & 22 & 1 & 10 & 0 & 0 \\
\hline \multicolumn{9}{|l|}{ Headache on the day of treatment } \\
\hline Mild & 2 & 7 & 1 & 11 & 0 & 0 & 1 & 11 \\
\hline Middle & 10 & 36 & 4 & 44 & 5 & 50 & 1 & 11 \\
\hline Intense & 1 & 4 & 1 & 11 & 0 & 0 & 0 & 0 \\
\hline \multicolumn{9}{|l|}{ Headache on following days } \\
\hline Mild & 3 & 11 & 2 & 22 & 1 & 10 & 0 & 0 \\
\hline Middle & 1 & 4 & 1 & 11 & 0 & 0 & 0 & 0 \\
\hline Intense & 1 & 4 & 1 & 11 & 0 & 0 & 0 & 0 \\
\hline Paresthesia or pain of lower limb & 10 & 36 & 4 & 44 & 3 & 30 & 3 & 33 \\
\hline Paresthesia of upper limb & 6 & 21 & 1 & 11 & 3 & 30 & 2 & 22 \\
\hline Increased bladder spasticity & 1 & 4 & 1 & 11 & 0 & 0 & 0 & 0 \\
\hline Unspecific facial pain & 4 & 14 & 0 & 0 & 3 & 30 & 1 & 11 \\
\hline Restless legs/spasticity over night & 3 & 11 & 2 & 22 & 0 & 0 & 1 & 11 \\
\hline Gait disturbance & 2 & 7 & 0 & 0 & 1 & 10 & 1 & 11 \\
\hline Dizziness & 1 & 4 & 0 & 0 & 1 & 10 & 0 & 0 \\
\hline Tiredness on the day following treatment & 2 & 7 & 0 & 0 & 0 & 0 & 2 & 22 \\
\hline Dorsal pain & 1 & 4 & 0 & 0 & 1 & 10 & 0 & 0 \\
\hline Unspecific feeling of discomfort & 2 & 7 & 1 & 11 & 1 & 10 & 0 & 0 \\
\hline
\end{tabular}

Abbreviations: $\mathrm{MC}=$ motor cortex; $\mathrm{PFC}=$ prefrontal cortex. frequency of each group was too low to allow for comparative analysis (table 2), and all patients fully recovered from AEs within a few days.

All 5 premature dropouts occurred during the treatment phase: 1 due to intensification of known trigeminal neuralgia, 3 (1 from each treatment group) due to scalp discomfort or headache during treatment (and, in 1 case, due to time constraints after the initial four treatments), and, last, 1 patient because of claustrophobia experienced under the stimulation device.

Exploratory clinical efficacy. Twenty-seven of 28 patients (96.4\%) were classified as fatigued at BL with an FSS score of 4.0 or more (9 patients per group, table 1), of which $7(7 / 27=25.9 \%)$ were nonfatigued at the final FU (3 sham-, 1 PFC-, and 3 MC-stimulated patients). The single nonfatigued patient at $\mathrm{BL}(\mathrm{FSS}=2.8)$ was subsequently assessed as fatigued (FSS $=5.7$ ) after PFC sham stimulation (see also figure e-1, http://links.lww. com/NXI/A2 for individual data curves). There was no significant FSS difference at BL between the treatment groups (table 1).

All patients with fatigue at BL showed significant improvement after treatment (FU1, figure 2). Calculated at the group level, the median FSS score decreased by 1.0 points (BL vs FU1, 95\% CI $0.45-$ 1.65; time effect: $\mathrm{df}=1.0, p<0.001$ ). This improvement was most evident in the MC group (1.74 points, $95 \%$ CI $0.41-2.95)$ compared with the PFC sham group $(0.77$ points, $95 \%$ CI 0.10 $2.30)$ and the PFC group $(0.35,95 \% \mathrm{CI}-0.35$ to 1.70). However, FSS scores did not differ between treatment groups at $\mathrm{BL}$ or FU (FU1) (median FSS scores at FU1 [IQR] 6.3 [4.7-6.7] PFC group; 5.2 [3.9-5.6] PFC sham group; 4.4 [3.8-4.7] MC stimulation; nonparametric ANOVA-like analysis of $\mathrm{BL}$ vs FU1 group effect: $\mathrm{df}=1.8, p=0.279$ ). In addition, FSS scores were still significantly lower 4 weeks later, at FU3 compared with BL (median decrease of the FSS score of 1.1 points, $95 \%$ CI $0.55-1.68$; time effect: $\mathrm{df}=1.0, p<0.001$, group effect: $\mathrm{df}=1.9$, $p=0.260$ ), with the MC group (1.78 points, $95 \%$ CI $0.85-2.75)$ continuing to show more improvement compared with the PFC group (0.75, 95\% CI -0.20 to 1.94$)$ and PFC sham (0.41 points, $95 \% \mathrm{CI}-0.35$ to 1.8 ) group (interaction group $\times$ time $\mathrm{df}=2.0, p=0.037)$. Indeed, the fatigue score did not change significantly between FU1 and FU3 over all groups (median difference $<0.01$ points, $95 \%$ CI -0.40 to 0.35 ), and there were no significant differences in the changes over time between groups (group $\times$ time, $\mathrm{df}=1.9, p=0.073$; figure 2). EDSS and MSFC showed no significant change over time or between groups (data not shown). We obtained a significant impact of treatment group on FSS scores at FU1 $(p=0.001)$ and also a significant 
Figure 2 Changes in fatigue under treatment

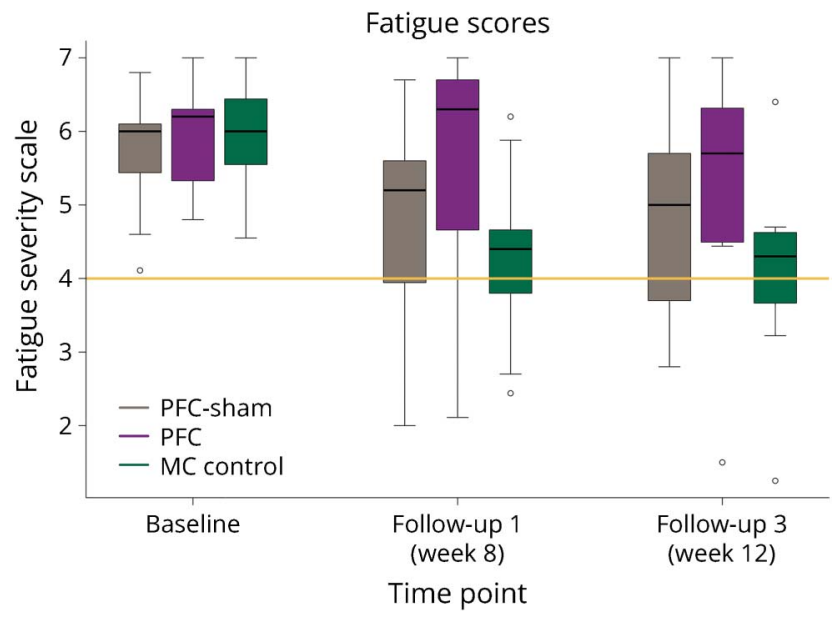

FSS changes during the study using standard boxplots. Treatment group "PFC" is shown in purple, treatment group "MC" in green, and sham group "PFC sham" in gray. Post-treatment visits are termed "follow-up" (plus follow-up visit number). The yellow line indicates FSS cutoff between fatigued $(F S S \geq 4)$ and nonfatigued values. FSS = Fatigue Severity Scale; $\mathrm{MC}=$ motor cortex; $\mathrm{PFC}=$ prefrontal cortex.

impact of FSS at BL $(p=0.015)$, but not of BDI at $\mathrm{BL}(p=0.848)$ in nonparametric (rank-based) ANOVA-like analysis, confirming our findings without these covariates.

DISCUSSION This randomized, sham-controlled phase I/IIa study applied stimulation of deeper brain regions by means of interventional $\mathrm{H}$-coil rTMS to patients with MS. We found that rTMS is safe in MS, and preliminary efficacy data further support investigating rTMS for symptomatic treatment of fatigue in MS. Approximately one-third of patients suffered from mild-to-moderate headache, which was the most frequently experienced adverse event, all of which were fully resolved within several days. The adverse events were distributed equally across treatment groups. In line with other clinical studies using rTMS, transient headache and scalp discomfort were the most frequently reported side effects. ${ }^{28}$ Fifteen percent of patients discontinued before completion of the study, with the majority (3 of 5 patients) reporting scalp discomfort and headache as reasons. These data are instructive for the design of future trials, particularly for the calculation of sample size and obtaining the consent of study participants.

Our exploratory analysis showed that rTMS significantly reduced fatigue in our cohort of $27 \mathrm{pa}-$ tients. This effect was most pronounced in the MC stimulation group, with a median decrease of 1.74 points in the FSS score directly after treatment compared with BL, which should be considered clinically meaningful. ${ }^{29}$ While the results are promising, they have to be interpreted with caution, given the small sample size and the exploratory nature of the study and of the efficacy analysis.

$\mathrm{H}$-coil enables nonfocal stimulation of deeper neuronal structures as compared to standard figure-of-eight coils. ${ }^{13,14}$ This may be an advantage when the therapeutic approach aims at targeting larger brain regions that are presumably involved in the pathophysiology of MDD (e.g., the lateral PFC, including the broader dorsolateral and ventrolateral PFC areas and their projections into subcortical networks ${ }^{18}$ ). Recent concepts propose that fatigue in MS is a network disorder associated with impaired functional connectivity. These connectivity alterations are thought to result from focal or diffuse tissue damage, with altered microstructural integrity of white matter tracts and gray matter. ${ }^{8,30,31}$ Disease-related disruption of interconnections between critical anatomical regions, such as the basal ganglia with the PFC, the posterior cingulate cortex, or cortical motor areas, may result in profoundly altered striatocortical connectivity. This, in turn, may impair motor (e.g., planning and execution of movements) and nonmotor (e.g., motivation and reward processing) functions, thus contributing to the pathophysiology of fatigue in MS. ${ }^{8,31}$ The network theory of MS fatigue is supported by numerous structural and functional neuroimaging studies and electrophysiologic investigations. ${ }^{8,32,33}$ As these regions are within the reach of the $\mathrm{H}$-coil, it is conceivable that deep TMS may at least in part and temporarily normalize the impaired functional connectivity and thus improve fatigue-similar to mechanisms thought to be involved in the reduction of depressive symptoms in MDD following rTMS. ${ }^{18}$

Although we found improvement in fatigue in both verum stimulation conditions, the decrease in fatigue severity was more pronounced following bilateral MC stimulation. Given the broad coverage of the $\mathrm{H}$-coil, the MC stimulation paradigm may possibly have influenced the activity of additional brain regions beyond motor areas. However, by generating a facilitatory input on primary motor and supplementary MC areas, as has been previously demonstrated for rTMS at $5 \mathrm{~Hz},{ }^{11}$ our stimulation paradigm may have ameliorated impaired recruitment patterns of supplementary motor areas, which are associated with fatigue. ${ }^{9}$

In contrast to previous studies, acute rTMS treatment in our study was not followed by a maintenance protocol..$^{18}$ Despite this, FSS did not return to BL levels during the entire 6-week FU phase, which is in line with reported sustained long-term effects for approximately 5 months in patients with MDD in an open label study. ${ }^{34}$ The sustained effect without the maintenance protocol suggests that clinical rTMS protocols for MS fatigue could involve fewer stimulation sessions ${ }^{18}$ following an initial induction phase, thereby reducing patients' burden of frequent visits to the clinic. 
This study prioritized the assessment of safety and tolerability as a primary end point at the expense of investigating preliminary efficacy. Thus, all data on the effects of rTMS on MS-associated fatigue are preliminary and should be interpreted with caution. Placebo effects of rTMS may also lead to a relevant reduction of symptoms as observed in previous rTMS studies. ${ }^{35}$ Furthermore, as our sham condition used the stimulation parameters of active PFC rTMS, but not MC, potential sensory side effects of sham (for example, alertness caused by auditory stimuli such as the frequency of the clicking sound) were controlled for PFC, but not for MC. To confirm our data, further studies should use stimulation parameters adapted to sham control for MC stimulation.

Fatigue can affect cognitive and motor aspects differently, which lead to the conceptualization of cognitive fatigue and motor fatigue as potentially independent constructs. Thus, some instruments, e.g., the Modified Fatigue Impact Scale (MFIS) differentiates motor, cognitive, and psychosocial fatigue in its subscales. ${ }^{36}$ The FSS scale used in our study does not differentiate between cognitive and motor aspects, but treats fatigue as its own entity, which can affect both motor and cognitive tasks. ${ }^{21}$ Although cognitive aspects are explicitly covered only in 1 question in the FSS, the total FSS score appropriately correlates with both cognitive and motor fatigue subscales of the MFIS, which is why the results of our study should be applicable for both motor and cognitive aspects of fatigue. ${ }^{37}$ Nonetheless, it will be very interesting to apply an instrument like the Fatigue Scale for Motor and Cognitive functions, which is more appropriate to dissect cognitive and motor fatigue, in a future Phase IIb or III study. ${ }^{38}$

Finally, the study design included depression as assessed by the BDI-IA as further exploratory end point. However, after enrollment completion, the distribution of patients classified as depressed was uneven between the groups, which is why we abstained from further analyzing effects on depressive symptoms in this study.

In light of the safety and tolerability of rTMS treatment in MS-associated fatigue, our study strongly suggests further investigating its potential therapeutic efficacy and the underlying mechanisms.

\section{ACKNOWLEDGMENT}

The authors thank Katharina Stößlein (NeuroCure Cluster of Excellence, Charité-Universitätsmedizin Berlin) for support in organization and execution of the study. They also thank Amit Ginou (Brainsway Ltd., Jerusalem) for technical support. James Mateo Ari Liebkowsky edited the manuscript for nonintellectual content.

\section{STUDY FUNDING}

No targeted funding reported.

\section{DISCLOSURE}

G. Gaede, M. Tiede, and I. Lorenz report no disclosures. A.U. Brandt served on the scientific advisory board of Biogen; received travel funding and/or speaker honoraria from Novartis, Biogen, and Teva; has patents pending for perceptive visual computing based postural control analysis, multiple sclerosis biomarker, perceptive sleep motion analysis, and fovea morphometry; consulted for Novartis and Biogen; received research support from BMWi, BMBF, and Guthy Jackson Charitable Foundation; and holds stock or stock options in Motognosis. C. Pfueller reports no disclosures. J. Dörr served on the scientific advisory board of Bayer, Novartis, Teva, and Sanofi-Genzyme; received travel funding and/or speaker honoraria from Novartis, Sanofi-Genzyme, Biogen, Bayer, Teva, and Allergan; and received research support from Novartis and Bayer. J. Bellmann-Strobl received speaker honoraria and travel funding from Bayer, Sanofi-Aventis/ Genzyme, Merck, and Teva. S.K. Piper is a statistical reviewer for the Journal of Cerebral Blood Flow and Metabolism. Y. Roth is employed by Brainsway Ltd. A. Zangen served on the scientific advisory board of Brainsway, a company that produces the H-coils for deep TMS (including the ones used in the current study); served as an editorial advisory board member for the journal Brain Stimulation; holds a patent for first inventor for H-coils for deep transcranial magnetic stimulation; consulted for Brainsway, Brainsway might gain profit if the current published article will end up being a basis for clinical practice in the treatment of multiple sclerosis in the future; received research support from the NIH, the Israel Science Foundation, the Binational Science Foundation, and the BrightFocus Foundation; holds stocks in Brainsway; receives royalties from the $\mathrm{NIH}$ and the Weizmann Institute of Science; and participated in legal proceedings in Brainway. S. Schippling served on the scientific advisory board of Bayer, Biogen, Merck Serono, Novartis, Sanofi-Genzyme, and Teva; received travel funding and/or speaker honoraria from Bayer, Biogen, Merck Serono, Novarits, Sanofi-Genzyme, and Teva; served as an associate editor for Frontiers in Neurology; holds a patent in therapeutic vaccine in PML using VP1 and Il7; and received research support from SanofiGenzyme, Novartis, the University of Zurich, the Betty and David Koetser Foundation for Brain Research, and the Swiss Multiple Sclerosis Society. F. Paul served on the scientific advisory board of Novartis; received speaker honoraria and travel funding from Bayer, Novartis, Biogen, Teva, SanofiAventis/Genzyme, Merck Serono, Alexion, Chugai, MedImmune, and Shire; served as an academic editor for PLoS One; is an associate editor for Neurology ${ }^{\circledR}$ Neuroimmunology \& Neuroinflammation; consulted for Sanofi-Genzyme, Biogen, MedImmune, Shire, and Alexion; received research support from Bayer, Novartis, Biogen, Teva, Sanofi-Aventis/ Genzyme, Alexion, Merck Serono, German Research Council, Werth Stiftung of the City of Cologne, German Ministry of Education and Research, Arthur Arnstein Stiftung Berlin, Arthur Arnstein Foundation Berlin, Guthy Jackson Charitable Foundation, and National Multiple Sclerosis Society of the United States. Go to Neurology.org/nn for full disclosure forms.

\section{AUTHOR CONTRIBUTIONS}

Mr. Gaede: acquisition of data, interpretation of data, study supervision, and critical revision of the manuscript for intellectual content. Ms. Tiede and Ms. Lorenz: acquisition of data and interpretation of data. Dr. Brandt: analysis and interpretation of data and critical revision of the manuscript for intellectual content. Mr. Pfueller, Dr. Dörr, and Dr. Bellmann-Strobl: acquisition of data, interpretation of data, and study supervision. Dr. Piper: analysis and interpretation of data. Dr. Roth and Prof. Zangen: critical revision of the manuscript for intellectual content. Dr. Schippling and Prof. Paul: study concept and design and critical revision of the manuscript for intellectual content.
Received April 28, 2017. Accepted in final form September 6, 2017.

\section{REFERENCES}

1. Compston A, Coles A. Multiple sclerosis. Lancet 2008; 372:1502-1517.

2. Krupp L. Fatigue is intrinsic to multiple sclerosis (MS) and is the most commonly reported symptom of the disease. Mult Scler 2006;12:367-368.

3. Mitchell AJ, Benito-León J, González JMM, Rivera-Navarro J. Quality of life and its assessment in multiple sclerosis: 
integrating physical and psychological components of wellbeing. Lancet Neurol 2005;4:556-566.

4. Smith MM, Arnett PA. Factors related to employment status changes in individuals with multiple sclerosis. Mult Scler 2005;11:602-609.

5. Bonavita S, Tedeschi G, Gallo A. Morphostructural MRI abnormalities related to neuropsychiatric disorders associated to multiple sclerosis. Mult Scler Int 2013;2013:104254.

6. Nygaard GO, Walhovd KB, Sowa P, et al. Cortical thickness and surface area relate to specific symptoms in early relapsingremitting multiple sclerosis. Mult Scler 2015;21:402-414.

7. Damasceno A, Damasceno BP, Cendes F. Atrophy of reward-related striatal structures in fatigued MS patients is independent of physical disability. Mult Scler 2016;22: 822-829.

8. Finke C, Schlichting J, Papazoglou S, et al. Altered basal ganglia functional connectivity in multiple sclerosis patients with fatigue. Mult Scler 2015;21:925-934.

9. Rocca MA, Meani A, Riccitelli GC, et al. Abnormal adaptation over time of motor network recruitment in multiple sclerosis patients with fatigue. Mult Scler 2016;22:1144-1153.

10. McNamara B, Ray JL, Arthurs OJ, Boniface S. Transcranial magnetic stimulation for depression and other psychiatric disorders. Psychol Med 2001;31:1141-1146.

11. Centonze D, Koch G, Versace V, et al. Repetitive transcranial magnetic stimulation of the motor cortex ameliorates spasticity in multiple sclerosis. Neurology 2007;68: 1045-1050.

12. Centonze D, Petta F, Versace V, et al. Effects of motor cortex rTMS on lower urinary tract dysfunction in multiple sclerosis. Mult Scler 2007;13:269-271.

13. Zangen A, Roth Y, Voller B, Hallett M. Transcranial magnetic stimulation of deep brain regions: evidence for efficacy of the H-Coil. Clin Neurophysiol 2005;116:775-779.

14. Roth Y, Amir A, Levkovitz Y, Zangen A. Three-dimensional distribution of the electric field induced in the brain by transcranial magnetic stimulation using figure- 8 and deep H-coils. J Clin Neurophysiol 2007;24:31-38.

15. Levkovitz Y, Roth Y, Harel EV, Braw Y, Sheer A, Zangen A. A randomized controlled feasibility and safety study of deep transcranial magnetic stimulation. Clin Neurophysiol 2007;118:2730-2744.

16. Levkovitz Y, Harel EV, Roth Y, et al. Deep transcranial magnetic stimulation over the prefrontal cortex: evaluation of antidepressant and cognitive effects in depressive patients. Brain Stimul 2009;2:188-200.

17. Rosenberg O, Shoenfeld N, Zangen A, Kotler M, Dannon PN. Deep TMS in a resistant major depressive disorder: a brief report. Depress Anxiety 2010;27:465-469.

18. Levkovitz Y, Isserles M, Padberg F, et al. Efficacy and safety of deep transcranial magnetic stimulation for major depression: a prospective multicenter randomized controlled trial. World Psychiatry 2015;14:64-73.

19. Bakshi R, Shaikh ZA, Miletich RS, et al. Fatigue in multiple sclerosis and its relationship to depression and neurologic disability. Mult Scler 2000;6:181-185.

20. Polman $\mathrm{CH}$, Reingold SC, Edan G, et al. Diagnostic criteria for multiple sclerosis: 2005 revisions to the "McDonald Criteria." Ann Neurol 2005;58:840-846.

21. Krupp LB, LaRocca NG, Muir-Nash J, Steinberg AD. The fatigue severity scale: application to patients with multiple sclerosis and systemic lupus erythematosus. Arch Neurol 1989;46:1121-1123.
22. Beck AT, Steer RA. Internal consistencies of the original and revised Beck depression inventory. J Clin Psychol 1984;40:1365-1367.

23. Weinges-Evers N, Brandt AU, Bock M, et al. Correlation of self-assessed fatigue and alertness in multiple sclerosis. Mult Scler 2010;16:1134-1140.

24. Kurtzke JF. Rating neurologic impairment in multiple sclerosis: an expanded disability status scale (EDSS). Neurology 1983;33:1444-1452.

25. MSFC Manual [online]. Available at: main. nationalmssociety.org/docs/HOM/MSFC_Manual_and_ Forms.pdf. Accessed April 18, 2016.

26. Rossini PM, Barker AT, Berardelli A, et al. Non-invasive electrical and magnetic stimulation of the brain, spinal cord and roots: basic principles and procedures for routine clinical application. Report of an IFCN committee. Electroencephalogr Clin Neurophysiol 1994;91:79-92.

27. Ihaka R, Gentleman R. R: a language for data analysis and graphics. J Comput Graph Stat 1996;5:299-314.

28. Bersani FS, Minichino A, Enticott PG, et al. Deep transcranial magnetic stimulation as a treatment for psychiatric disorders: a comprehensive review. Eur Psychiatry 2013; 28:30-39.

29. Lange R, Volkmer M, Heesen C, Liepert J. Modafinil effects in multiple sclerosis patients with fatigue. J Neurol 2009;256:645-650.

30. Calabrese M, Rinaldi F, Grossi P, et al. Basal ganglia and frontal/parietal cortical atrophy is associated with fatigue in relapsing-remitting multiple sclerosis. Mult Scler 2010;16: $1220-1228$.

31. Chaudhuri A, Behan PO. Fatigue and basal ganglia. J Neurol Sci 2000;179:34-42.

32. Filippi M, Rocca MA, Colombo B, et al. Functional magnetic resonance imaging correlates of fatigue in multiple sclerosis. Neuroimage 2002;15:559-567.

33. Leocani L, Colombo B, Magnani G, et al. Fatigue in multiple sclerosis is associated with abnormal cortical activation to voluntary movement-EEG evidence. Neuroimage 2001;13:1186-1192.

34. Demirtas-Tatlidede A, Mechanic-Hamilton D, Press DZ, et al. An open-label, prospective study of repetitive transcranial magnetic stimulation (rTMS) in the long-term treatment of refractory depression: reproducibility and duration of the antidepressant effect in medication-free patients. J Clin Psychiatry 2008;69:930-934.

35. Okabe S, Ugawa Y, Kanazawa I; Effectiveness of rTMS on Parkinson's Disease Study Group. 0.2-Hz repetitive transcranial magnetic stimulation has no add-on effects as compared to a realistic sham stimulation in Parkinson's disease. Mov Disord 2003;18:382-388.

36. Téllez N, Río J, Tintoré M, Nos C, Galán I, Montalban $\mathrm{X}$. Does the Modified Fatigue Impact Scale offer a more comprehensive assessment of fatigue in MS? Mult Scler 2005;11:198-202.

37. Amtmann D, Bamer AM, Noonan V, Lang N, Kim J, Cook KF. Comparison of the psychometric properties of two fatigue scales in multiple sclerosis. Rehabil Psychol 2012;57:159-166.

38. Penner IK, Raselli C, Stöcklin M, Opwis K, Kappos L, Calabrese P. The Fatigue Scale for Motor and Cognitive Functions (FSMC): validation of a new instrument to assess multiple sclerosis-related fatigue. Mult Scler 2009; 15:1509-1517. 


\title{
Neurology \\ Neuroimmunology \& Neuroinflammation
}

\author{
Safety and preliminary efficacy of deep transcranial magnetic stimulation in \\ MS-related fatigue \\ Gunnar Gaede, Marina Tiede, Ina Lorenz, et al. \\ Neurol Neuroimmunol Neuroinflamm 2018;5; \\ DOI 10.1212/NXI.0000000000000423
}

This information is current as of December 14, 2017

\section{Updated Information \& Services \\ Supplementary Material \\ References \\ Subspecialty Collections}

Permissions \& Licensing

Reprints including high resolution figures, can be found at:

http://nn.neurology.org/content/5/1/e423.full.html

Supplementary material can be found at:

http://nn.neurology.org/content/suppl/2018/01/04/5.1.e423.DC1

This article cites 37 articles, 0 of which you can access for free at: http://nn.neurology.org/content/5/1/e423.full.html\#\#ref-list-1

This article, along with others on similar topics, appears in the following collection(s):

Class III

http://nn.neurology.org//cgi/collection/class_iii

Multiple sclerosis

http://nn.neurology.org//cgi/collection/multiple_sclerosis

TMS

http://nn.neurology.org//cgi/collection/tms

Information about reproducing this article in parts (figures,tables) or in its entirety can be found online at:

http://nn.neurology.org/misc/about.xhtml\#permissions

Information about ordering reprints can be found online:

http://nn.neurology.org/misc/addir.xhtml\#reprintsus

Neurol Neuroimmunol Neuroinflamm is an official journal of the American Academy of Neurology.

Published since April 2014, it is an open-access, online-only, continuous publication journal. Copyright

Copyright (C) 2017 The Author(s). Published by Wolters Kluwer Health, Inc. on behalf of the American

Academy of Neurology. All rights reserved. Online ISSN: 2332-7812.

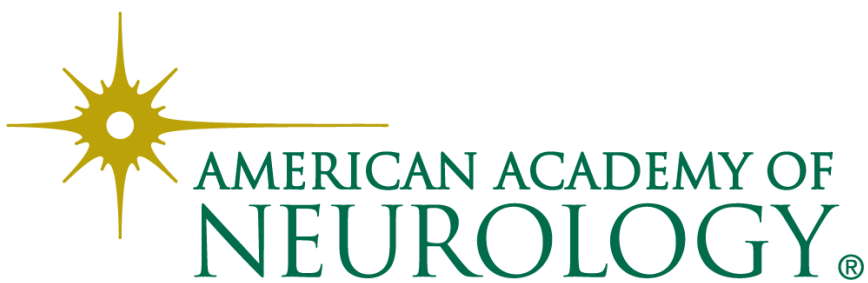

